\title{
THE HILBERT TRANSFORM OF SCHWARTZ DISTRIBUTIONS
}

\author{
J. N. PANDEY
}

\begin{abstract}
Let $\mathscr{D}$ be the Schwartz space of infinitely differentiable complex-valued functions defined on the real line with compact supports equipped with the usual topology. Assume $H(\mathbb{D})$ to be the space of $C^{\infty}$ functions defined on the real line whose every element is the Hilbert transform of an element of $\mathscr{D}$. We equip the space $H(D)$ with an appropriate topology and show that the classical Hilbert transformation $H$, defined by $H f=P \int_{-\infty}^{\infty} f(t) /(t-x) d t$, is a homeomorphism from $\mathcal{D}$ onto $H(\mathscr{D})$. The Hilbert transform $H f$ of $f \in \mathcal{D}^{\prime}$ is then defined to be an element of $H^{\prime}(\mathcal{D})$ given by the relation

$$
\langle H f, \varphi\rangle=\langle f,-H \varphi\rangle \forall \varphi \in H(\mathscr{D}) .
$$

It then follows that $-H^{2} f / \pi^{2}=f \forall f \in \mathbb{D}^{\prime}$.

Applications of our results in solving some singular integral equations are also discussed.
\end{abstract}

Introduction. The classical Hilbert transform

$$
H f=P \int_{-\infty}^{\infty} \frac{f(t)}{t-x} d t
$$

and its inversion formula $H^{2} f / \pi^{2}=f$ were proved by Titchmarsh and Riesz [8] for the space $L^{p}, p>1$, interpreting convergence in the $L^{p}$ sense. Since then various authors such as Beltrami and Wohlers [1], Gel'fand and Shilov [3], Orton [5], Mitrovic [4], etc. extended the Hilbert transform and its inversion formula to various subspaces of Schwartz distribution space ' $\mathcal{D}^{\prime}$. Most notable amongst them is the work done by Orton [5], who extended the Hilbert transform to an arbitrary element of $\mathcal{D}^{\prime}$. However, her definition of Hilbert transform of distributions is based upon the analytic representation of distributions and, as such, is not quite constructive, unless the distribution concerned happens to be of compact support or certain very familiar types of regular distributions. Besides, her technique is not very well suited to solving certain singular integral equations involving the Hilbert transform. Our objective is to extend the Hilbert transform and its inversion formula to an arbitrary element of $\mathscr{D}^{\prime}$ and indicate its applicability in solving singular integral equations in the space $\mathscr{D}^{\prime}$. Our method of extension is quite constructive and simple as well.

DEFINITIONS AND PRELIMINARIES. In the sequel we assume throughout that $p$ is a real number greater than 1 .

\footnotetext{
Received by the editors January 4, 1982 and, in revised form, November 1, 1982. This paper was presented to the Indian Science Congress meeting at Tirupati, India on January 6, 1983.

1980 Mathematics Subject Classification. Primary 46F12; Secondary 44A15.

Key words and phrases. Generalized integral transform, integral transform of distributions.
} 
The testing function space $\mathscr{D}_{L^{p}}$ : The testing function space $\mathscr{D}_{L^{p}}$ consists of $C^{\infty}$ functions defined on the real line which, along with its every derivative, belongs to $L^{p}$. The concept of convergence and completeness in $\mathscr{D}_{L^{p}}$ is defined in the usual way [7] with respect to the topology introduced on it. It is a known fact that $E^{\prime} \subset\left(\mathscr{Q}_{L^{p}}\right)^{\prime}$ $\subset \mathscr{D}^{\prime}$, where $E^{\prime}$ is the space of distributions with compact supports on the real line $[7,10]$. If $\varphi(x) \in \mathscr{D}_{L^{p}}$, then $\varphi(x)$ along with all its derivatives tends to zero as $x \rightarrow \pm \infty$ [7]. Further, if $\left\{\varphi_{\nu}(x)\right\}_{\nu=1}^{\infty}$ is a sequence in $\mathscr{D}_{L^{p}}$ tending to the zero element in $\mathscr{D}_{L^{p}}$, with respect to the topology of $\mathscr{Q}_{L^{p}}$, as $\nu \rightarrow \infty$, then for each $k=0,1,2, \ldots$, $\varphi_{\nu}^{(k)}(x) \rightarrow 0$ uniformly $\forall x \in R$ as $\nu \rightarrow+\infty$ [7].

The testing function space $H(\mathscr{D})$. A complex-valued $C^{\infty}$ function $\varphi$ defined on the real line belongs to the space $H(\mathscr{D})$ iff $\varphi(x)$ is the Hilbert transform of some element $\psi \in \mathscr{D}$. That is to say, $\varphi(x) \in H(\mathscr{D}) \Leftrightarrow$ there exists $\psi(t) \in \mathscr{D}$ such that

$$
\varphi(x)=P \int_{-\infty}^{\infty} \frac{\psi(t)}{t-x} d t=H \psi
$$

where the integral is being taken in the Cauchy principal-value sense. We transport the topology of $\mathscr{D}$ to the space $H(\mathscr{D})$ by means of the operator $H$ defined by (1), i.e. a sequence $\left\{\varphi_{\nu}\right\}_{\nu=1}^{\infty}$ in $H(\mathscr{D})$ is said to converge to zero in $H(\mathscr{D})$ as $\nu \rightarrow \infty$ iff the associated sequence $\left\{\psi_{\nu}\right\}_{\nu=1}^{\infty}$ in $\mathscr{D}$ (where $H \psi_{\nu}=\varphi_{\nu}$ ) tends to zero in $\mathscr{D}$ as $\nu \rightarrow \infty$.

We now prove Theorems 1 and 2, which will be used in the sequel, and whose generalization will appear elsewhere [6].

THEOREM 1. Let $\varphi(x) \in \mathscr{D}$ and define $\psi(x)$ by

$$
\psi(x)=P \int_{-\infty}^{\infty} \frac{\varphi(t)}{t-x} d t
$$

Then for each $k=1,2,3, \ldots$,

$$
\psi^{(k)}(x)=P \int_{-\infty}^{\infty} \frac{\varphi^{(k)}(t)}{t-x} d t
$$

Proof. We will prove the result for $k=1$; the general result can be similarly proved by induction. Using the transformation $t-x=y$, it can be shown that

$$
\psi(x)=P \int_{-\infty}^{\infty} \frac{\varphi(t+x)}{t} d t .
$$

Now,

$$
\begin{aligned}
\psi(x) & =P \int_{-1}^{1}\left[\frac{\varphi(t+x)}{t}-\frac{\varphi(x)}{t}\right] d t+\left(\int_{-\infty}^{-1}+\int_{1}^{\infty}\right) \frac{\varphi(t+x)}{t} d t \\
& =\int_{-1}^{1} \psi(t, x) d t+\left(\int_{-\infty}^{-1}+\int_{1}^{\infty}\right) \frac{\varphi(t+x)}{t} d t
\end{aligned}
$$

where

$$
\begin{array}{cl}
\psi(t, x)=\varphi(t+x) / t-\varphi(x) / t & \text { if } t \neq 0 \\
=\varphi^{\prime}(x) & \text { if } t=0 .
\end{array}
$$

Clearly, $\psi(t, x) \in C^{\infty}\left(R^{2}\right)$. 
Now, it is not difficult to see that

$$
\begin{aligned}
\psi^{(1)}(x) & =\int_{-1}^{1} \frac{\partial \psi}{\partial x} d t+\left(\int_{-\infty}^{-1}+\int_{1}^{\infty}\right) \frac{\varphi^{\prime}(t+x)}{t} d t \\
& =\operatorname{lt}_{\varepsilon \rightarrow 0+}\left(\int_{-1}^{-\varepsilon}+\int_{\varepsilon}^{1}\right) \frac{\partial \psi}{\partial x} d t+\left(\int_{-\infty}^{-1}+\int_{1}^{\infty}\right) \frac{\varphi^{\prime}(t+x)}{t} d t \\
& =P \int_{-1}^{1}\left[\frac{\varphi^{\prime}(t+x)}{t}-\frac{\varphi^{\prime}(x)}{t}\right] d t+\left(\int_{-\infty}^{-1}+\int_{1}^{\infty}\right) \frac{\varphi^{\prime}(t+x)}{t} d t \\
& =P \int_{-\infty}^{\infty} \frac{\varphi^{\prime}(t+x)}{t} d t=P \int_{-\infty}^{\infty} \frac{\varphi^{\prime}(t)}{t-x} d t .
\end{aligned}
$$

THEOREM 2. Let $H(\mathscr{D})$ and $\mathscr{D}_{L^{p}}$ be the spaces defined as before. Then:

(i) $H(\mathscr{D}) \subset \mathscr{D}_{L^{p}}$ and $H(\mathscr{D})$ is dense in $\mathscr{D}_{L^{p}}$.

(ii) Convergence of a sequence in $H(\mathscr{D})$ implies its convergence in $\mathscr{D}_{L^{p}}$.

Proof. (i) Let $\varphi$ be an element of $\mathscr{D}$ with support contained in the closed interval $[-a, a]$. Then by Theorem 1 we have

$$
(H \varphi)^{(k)}(x)=P \int_{-a}^{a} \frac{\varphi^{(k)}(t)}{t-x} d t
$$

Therefore,

$$
(H \varphi)^{(k)}(x)=O(|\ln |(x+a) /(x-a)||) \text { as }|x| \rightarrow \infty .
$$

Hence, if $\psi$ is an element of $H(\mathscr{D})$ satisfying $\psi=H \varphi$, then $\psi^{(k)}(x)=O(1 /|x|)$ as $|x| \rightarrow \infty$. Therefore, $\psi(x) \in \mathscr{D}_{L^{p}}$. In view of the inversion formula [8]

$$
-H^{2} f / \pi^{2}=f \quad \forall f \in L^{p}
$$

it follows that for $\psi \in \mathscr{D}_{L^{p}}$ there exists $\theta(x) \in \mathscr{D}_{L^{p}}$ such that

$$
(H \theta)(x)=\psi \text {. }
$$

Since $\mathscr{D}$ is dense in $\mathscr{D}_{L^{p}}\left[7\right.$, pp. 199-200], there exists a sequence $\left\{\theta_{\nu}\right\}_{\nu=1}^{\infty}$ in $\mathscr{D}$ tending to $\theta$ in $\mathscr{D}_{L^{p}}$ as $\nu \rightarrow \infty$. Now,

$$
\left\|\frac{d^{k}}{d x^{k}}\left(\psi(x)-\left(H \theta_{\nu}\right)(x)\right)\right\|_{p}=\left\|H\left(\theta^{(k)}-\theta_{\nu}^{(k)}\right)\right\|_{p} \leqslant c_{p}\left\|\theta^{(k)}-\theta_{\nu}^{(k)}\right\|_{p} \rightarrow 0 \text { as } \nu \rightarrow \infty
$$

(Theorem 1 and [8, pp. 132-133]). Therefore, $H(\mathscr{D})$ is dense in $\mathscr{Q}_{L^{p}}$. This completes the proof of (i).

(ii) If $\left\{\varphi_{\nu}\right\}$ is a sequence in $H(\mathscr{D})$ converging to zero in $H(\mathscr{D})$ as $\nu \rightarrow \infty$, then there exists a sequence $\left\{\psi_{\nu}\right\}$ in $\mathscr{D}$ tending to zero in $\mathscr{D}$ as $\nu \rightarrow \infty$ such that $H \psi_{\nu}=\varphi_{\nu}$. Using Theorem 1 and [8, pp. 132-133], we have $\left\|\varphi_{\nu}^{(k)}\right\|_{p} \leqslant c_{p}\left\|\psi_{\nu}^{(k)}\right\|_{p} \rightarrow 0$ as $\nu \rightarrow \infty$.

REMARK 1. Theorem 2, in fact, proves that the space $H^{\prime}(\mathscr{D})$ of ultradistributions consisting of continuous linear functionals on $H(\mathscr{D})$ contains the space $\left(\mathscr{D}_{L^{p}}\right)^{\prime}$.

REMARK 2. In view of the inversion formula (5), it can be seen that $H$ is a linear homeomorphism from $\mathscr{D}$ onto $H(\mathscr{D})$ and the mapping $H: H(\mathscr{D}) \rightarrow \mathscr{D}$ is linear and continuous. 
Generalized Hilbert transformation. The generalized Hilbert transform $H f$ of $f \in \mathscr{Q}^{\prime}$ is defined to be an ultradistribution $H f \in H^{\prime}(\mathcal{O})$ such that

$$
\langle H f, \varphi\rangle=\langle f,-H \varphi\rangle \quad \forall \varphi \in H(\mathcal{D}) \text {, }
$$

where $H \varphi$ is the classical Hilbert transform defined by (1). If $g \in H^{\prime}($ O $)$, its Hilbert transform $\mathrm{Hg}$ is defined to be a Schwartz distribution by the relation

$$
\langle H g, \varphi\rangle=\langle g,-H \varphi\rangle \quad \forall \varphi \in \mathbb{Q} \text {. }
$$

From (5), (7) and (8) it follows that $\left\langle-H^{2} f / \pi^{2}, \varphi\right\rangle=\langle f, \varphi\rangle \forall \varphi \in \mathscr{Q}$, i.e.

$$
H^{2} f / \pi^{2}=f \quad \forall f \in \mathcal{O Q}^{\prime} .
$$

It may be noted that the definition (7) of the generalized Hilbert transform is in agreement with the classical result proved in $[9, \mathrm{p}$. 170]. It is a simple corollary to show that if $f \in L^{p}$ then the generalized Hilbert transform $H f$ of the regular generalized function $f$ generated by $f(t)$ is a regular ultradistribution generated by $P \int_{-\infty}^{\infty} f(t) /(t-x) d t$.

Definition. The derivative $g^{\prime}$ of an ultradistribution $g \in H^{\prime}(\mathcal{D})$ is defined to be an ultradistribution belonging to $H^{\prime}(\mathcal{O})$ ) by the relation

$$
\left\langle g^{\prime}, \varphi\right\rangle=\left\langle g,-\varphi^{\prime}\right\rangle \quad \forall \varphi \in H\left(\mathcal{O}^{\prime}\right) \text {. }
$$

THEOREM 3. Let $f \in \mathcal{O D}^{\prime}$, then

$$
(H f)^{(k)}=H\left(f^{(k)}\right) \text { for each } k=1,2,3, \ldots,
$$

where $(H f)^{k}$ stands for the kth derivative of the ultradistribution $H f$.

Proof. We will prove the result for $k=1$, and the general result can be similarly proved by induction. For $\varphi \in H\left({ }^{(D)}\right)$ we have

$$
\begin{aligned}
\left\langle H f^{\prime}, \varphi\right\rangle & =\left\langle f^{\prime},-H \varphi\right\rangle=\langle f, d H \varphi / d x\rangle \\
& =\langle f, H d(\varphi(t)) / d t\rangle \quad[\text { Theorem 1] } \\
& =\langle-H f, d(\varphi(t)) / d t\rangle=\left\langle(H f)^{\prime}, \varphi(t)\right\rangle \quad \forall \varphi \in H(\mathscr{D}) .
\end{aligned}
$$

This completes the proof of Theorem 3.

Using definition (7) it follows immediately that $H \delta=-P V(1 / x)$, and using the inversion formula (9) it follows that $H(P V / x)=\pi^{2} \delta$.

EXAMPLE 1. Let us find the distribution $y$ satisfying the singular integral equation

$$
\frac{d y}{d x}+P \int_{-\infty}^{\infty} \frac{f^{\prime}(t)}{t-x} d t=\delta(x), \quad f, f^{\prime} \in L^{p} .
$$

(10) can be rewritten as

$$
d y / d x+H f^{\prime}=\delta(x)
$$

or

$$
d(y+H f) / d x=\delta(x), \quad y+H f=h(x)+c,
$$

or

$$
y=h(x)+c-P \int_{-\infty}^{\infty} \frac{f(t)}{t-x} d t
$$


EXAMPLE 2. Let us now find a distribution $y(t)$ satisfying the (operator) integral equation

$$
y+H y=f
$$

where $f$ and $H f \in \mathscr{Q}^{\prime}$.

Operating on both sides of (11) by $H$ we have

$$
H y+H^{2} y=H f, \quad H y-\pi^{2} y=H f, \quad f-y-\pi^{2} y=H f, \quad y=\frac{f-H f}{\pi^{2}+1} .
$$

An open problem. It is an interesting, but hard, problem to give an intrinsic definition of the space $H(\mathscr{D})$ and its topology, a solution of which will lead to far reaching consequences.

ACKNOWLEDGEMENT. This work was supported by NSERC grant \# A5298.

\section{REFERENCES}

1. E. J. Beltrami and M. R. Wohlers, Distributional boundary value theorems and Hilbert transforms, Arch. Rational Mech. Anal. 18 (1965), 304-309.

2. Avner Friedman, Generalized functions and partial differential equations, Prentice-Hall, Englewood Cliffs, N. J., 1963.

3. I. M. Gel'fand and G. E. Shilov, Generalized functions, Vol. II, Academic Press, New York, 1968.

4. Dragisa Mitrovic, $A$ Hilbert boundary-value problem, Math. Balkanica 1 (1971), 177-180.

5. Marion Orton, Hilbert transforms, Plemelj relations and Fourier transforms of distributions, SIAM J. Math. Anal. 4 (1973), 656-667.

6. J. N. Pandey and A. Chaudhary, Hilbert transform of generalized functions and applications, Canad. J. Math. (to appear)

7. L. Schwartz, Theorie des distributions, Hermann, Paris, 1966.

8. E. C. Titchmarsh, Introduction to the theory of Fourier integrals, Oxford Univ. Press, 1967.

9. F. Tricomi, Integral equations, Interscience, New York, 1957.

10. A. H. Zemanian, Distribution theory and transform analysis, McGraw-Hill, New York, 1965.

Department of Mathematics and Statistics, Carleton University, Ottawa, Ontario, Canada K1S 5B6 